\title{
UTENSÍLIOS SANITÁRIOS: COMPARAÇÃO ENTRE PROCESSOS DE LIMPEZA E DESINFECÇÃO MANUAL E AUTOMATIZADO
}

Sanitary appliance: comparative study of automated and manual cleaning and disinfection processes

Utensilios sanitarios: comparación entre procesos de limpieza y desinfección manual y automatizado

Carmen Eulalia Pozzer ${ }^{*}$ (D), Marcia Arsego² (D), Ivana Gottardo Rocha3 ${ }^{(D)}$, Heloisa Helena Karnas Hoefel ${ }^{4}$ (D), Cinara Maisonette Duarte ${ }^{5}$ (D), Angelica Peres do Amaral ${ }^{6}$ (D), Rita Catalina Aquino Caregnato ${ }^{7}$ (D)

RESUMO: Objetivo: Comparar os resultados dos processos de limpeza e desinfecção manual e automatizado de Utensílios Sanitários (US). Método: Estudo experimental, descritivo, realizado por meio de culturas microbiológicas de US usados por pacientes acamados em uma unidade de internação de um hospital localizado no sul do Brasil. As amostras microbiológicas foram coletadas após limpeza e desinfecção, sendo 11 amostras de cada um dos três processos testados: automatizado, manual sem supervisão e manual com supervisão. Resultados: No processo automatizado, não houve crescimento de microrganismos patogênicos de relevância epidemiológica. No manual, em ambos processamentos realizados, conforme protocolo estabelecido pela instituição com e sem supervisão, houve crescimento de microrganismos patogênicos. Conclusão: Com base nos resultados obtidos no estudo, o método automatizado demonstrou ser seguro para utilização dos US na assistência à saúde. Os resultados obtidos no método manual não conferem segurança. Sugere-se que sejam realizados estudos com conhecimento prévio do grau de contaminação controlada por meio de cepas de referência.

Palavras-chave: Segurança do paciente. Aparelho sanitário. Desinfecção. Equipamentos e provisões hospitalares.

ABSTRACT: Objective: To compare the results of manual and automated cleaning and disinfection of Sanitary Appliance (US). Method: A descriptive experimental study, carried out by means of microbiological cultures of appliance used by patients bedridden in a hospitalization unit of a hospital located in the south of Brazil. Thirty three samples were collected after the cleaning and disinfection processes were carried out, eleven for each of the three methods available: automated, manual with and without supervision for microbiological evaluation. Results: In the automated process, pathogenic microorganisms of epidemiological relevance was now here to be found in the experiment. In the manual, in both processes performed, according to protocol established by the Institution with and without supervision, there was growth of pathogenic microorganisms. Conclusion: Therefore, in this study we conclude that the automated method for cleaning and disinfection has been shown to be safer for use in healthcare. The results obtained in the manual method do not confer safety. It is suggested that studies be carried out with references trains with controlled contamination.

Keywords: Patient safety. Bathroom equipament. Disinfection. Equipment and supplies, hospital.

RESUMEN: Objectivo: Comparar los procesos de limpieza y desinfección manual y por médio de um equipo automático de Utensilios Sanitarios (US). Método: Estudio experimental descriptivo de medio de cultivos microbiológicos de US de los que hicieron uso enfermos en piso de internación de un hospital delsur de Brasil. Las muestras microbiológicas fueron recolectadas después de La limpieza y desinfección, de lãs cuales 11 muestras de cada uno

\footnotetext{
'Enfermeira; mestre em Ensino na Saúde. Coordenadora da Esterilização de Produtos para Saúde da Santa Casa de Misericórdia de Porto Alegre - Porto Alegre (RS), Brasil.

${ }^{2}$ Enfermeira do Controle de Infecção Hospitalar da Santa Casa de Misericórdia de Porto Alegre - Porto Alegre (RS), Brasil.

${ }^{3}$ Bióloga do Laboratório de Microbiologia da Santa Casa de Misericórdia de Porto Alegre - Porto Alegre (RS), Brasil.

«Enfermeira; doutora em Enfermagem. Professora da Escola de Enfermagem da Universidade Federal do Rio Grande do Sul - Porto Alegre (RS), Brasil.

${ }^{5}$ Enfermeira. Gerente hospitalar do Hospital São Francisco da Santa Casa de Misericórdia de Porto Alegre - Porto Alegre (RS), Brasil.

${ }^{6}$ Enfermeira da Santa Casa de Misericórdia de Porto Alegre - Porto Alegre (RS), Brasil.

${ }^{7}$ Doutora em Educação. Professora do Departamento de Enfermagem da Universidade Federal de Ciências da Saúde de Porto Alegre - Porto Alegre (RS), Brasil.

*Autor correspondente: pozzer@santacasa.org.br

Recebido: 15/09/2018 - Aprovado: 10/06/2019

DOI: $10.5327 / Z 1414-4425201900030002$
} 
de los tres procesos probados: automatico, manual sin supervisión y manual com supervisión. Resultados: Em El proceso com el equipo automatico, no hubo crecimiento de microorganismos patógenos de relevancia epidemiológica. Em los dos procesos manuales con y sin supervisión, conforme protocolo establecido por La institución, hubo crecimiento de microorganismos patógenos. Conclusión: Por los resultados obtenidos em ele studio, se concluye que el la limpeza e nel equipo automático ha demostrado seguridade para usar los US en cuidados a La salud. Se sugiere que se realicen estúdios conconocimiento prévio del grado de contaminación controlada por medio de cepas de referencia.

Palabras clave: Seguridad del paciente. Aparatos sanitarios. Desinfección. Equipos y suministros de hospitales.

\section{INTRODUÇÃ̃o}

Para a realização da assistência de enfermagem segura, são fundamentais as boas práticas e o adequado processamento de Produtos para Saúde (PPS). A tradicional classificação dos artigos, segundo o grau de criticidade, em críticos, semicríticos e não críticos, conforme a invasão dos artigos no corpo humano, é ainda utilizada mundialmente e citada em publicações direcionadas às práticas referentes ao controle e à prevenção das Infecções Relacionadas à Assistência de Saúde (IRAS) ${ }^{1}$. Os artigos não críticos, objeto deste estudo, são os que possuem contato com pele íntegra, exigindo, no mínimo, processo de limpeza ${ }^{1}$.

A limpeza tem como finalidade remover a matéria orgânica, reduzindo as Unidades Formadoras de Colônias (UFC). A exigência para o processamento de PPS não críticos é menor quanto ao tratamento que deve ser utilizado para o uso seguro, por ter contato apenas com a pele íntegra ${ }^{2,3}$.

O uso indiscriminado dos antimicrobianos contribuiu para o desenvolvimento de bactérias multirresistentes por meio da pressão seletiva. Dessa forma, aumentou-se igualmente a preocupação em adotar medidas preventivas, envolvendo materiais e ambientes que contribuam para o controle da transmissão desses microrganismos. Materiais que entram em contato apenas com pele íntegra, mas são reutilizados por pessoas distintas, merecem atenção após o uso, quando previamente contaminados com matéria orgânica passível de conter maior número de UFC.

Diversos PPS utilizados no cuidado aos pacientes nos Estabelecimentos de Assistência à Saúde (EAS) são reutilizados (após serem submetidos ao processo de limpeza e desinfecção) por diferentes pacientes. Assim sendo, podem ser veículos de agentes infecciosos se houver falhas no processo de limpeza e desinfecção. Os Utensílios Sanitários (US), como comadres e papagaios utilizados para coletar eliminações dos pacientes impossibilitados de utilizar o vaso sanitário, podem ser importante fonte de contaminação cruzada ${ }^{4}$. As fezes são compostas de matéria orgânica com grande quantidade de microrganismos, portanto os recipientes que as recebem devem ser processados, atendendo as boas práticas de processamento.

Dentre os microrganismos patogênicos estão as Enterobactérias (Escherichia coli enteropatogênica, Salmonella, Shigella, Citrobacter, Klebsiella, Serratia, Enterobacter, Proteus e Providencia), que causam infecções do trato urinário, entéricas, sistêmicas, bacteremias, pneumonias e meningites ${ }^{5}$.

Na limpeza manual dos US, realiza-se a friç̧ão mecânica com artefatos específicos, água corrente ou sob pressão e solução detergente, enquanto a limpeza automatizada é realizada por equipamentos utilizando jatos de água sob pressão e solução detergente ${ }^{6}$. Uma das desvantagens da limpeza manual dos US é o manuseio de excretas pelo trabalhador, ficando exposto ao risco de contaminação biológica. Embora na limpeza automatizada o contato com matéria orgânica também possa ocorrer, o risco é menor.

As lavadoras de US, denominadas também de lavadoras de descarga, permitem desprezar as excretas (fezes, urina, secreções e sangue), limpando e realizando a desinfecção de materiais não críticos, diminuindo o risco de infecções nos pacientes, risco ocupacional dos profissionais e impacto ambiental. São desenvolvidas para utilização nas unidades de internações, devendo ser instaladas em locais onde contenha expurgo e ligadas à rede elétrica, água e esgoto. Oferecem como vantagens: possibilidade de limpeza de mais de um produto ao mesmo tempo; otimização do recurso natural, como água; previne o risco ocupacional, biológico e químico; e realiza o processo de forma padronizada ${ }^{3}$. Para garantir o desempenho desse equipamento, o fabricante refere ser indispensável manter a intervenção preventiva e sua qualificação periódica, definida pelo EAS, sendo, no mínimo, anual ${ }^{6}$.

Com base nesta reflexão, traçou-se como problema de pesquisa: Qual é a diferença microbiana nos US quando realizado processo de limpeza e desinfecção automatizado e manual? 


\section{OBJETIVO}

Comparar os resultados dos processos de limpeza e desinfecção manual e automatizado em Utensílios Sanitários.

\section{MÉTODO}

Trata-se de um estudo experimental, descritivo, realizado em um EAS com 1.200 leitos, localizado na Região Sul do Brasil, em uma unidade de internação com 16 leitos.

O estudo foi realizado por meio de culturas microbiológicas dos US, após serem utilizados para eliminações fecais e urinárias de pacientes acamados na mesma unidade. A amostra foi constituída de 11 US para cada tipo de processo de limpeza e desinfecção, dos quais, após ter sido realizado o processo manual ou automatizado, foram coletadas culturas microbiológicas. As coletas das amostras foram realizadas pelo enfermeiro orientado pelo microbiologista, numeradas independente do processo de 1 a 33 e enviadas ao laboratório. Somente o coletador possuía a identificação do tipo de limpeza antes do resultado final da análise. Todas as coletas foram realizadas imediatamente após o término do processamento. O número médio de amostras foi estimado a partir de planilha de controle diário durante noventa dias de uso dos US com fezes e urina, utilizados na unidade em que se realizou o estudo.

Foram três os tipos de processos para limpeza e desinfecção dos US, conforme descrito a seguir:

- Manual sem supervisão do enfermeiro, com relato do técnico de enfermagem de ter realizado conforme Procedimento Operacional Padrão (POP): as excretas foram desprezadas na hidra do expurgo; a seguir, foi realizada a lavagem com detergente neutro hospitalar e escova específica para limpeza dos US, em água fria corrente; a secagem foi realizada com compressa limpa e desinfecção com compressa embebida em solução alcoólica a $70 \%$, por meio de fricção nas superfícies internas e externas. Esse procedimento foi repetido três vezes;

- Limpeza manual conforme POP: foi realizado o mesmo processo descrito no item anterior, porém com supervisão direta do enfermeiro;

- Limpeza automatizada: US recolhidos pelo técnico de enfermagem e introduzidos com excretas no rack no interior da lavadora, iniciando o comando automático; após a finalização do processo, o US foi retirado do equipamento; foi utilizada uma lavadora de descarga com as seguintes características: padrões para desinfecção térmica de temperatura entre $85 \mathrm{e}$ $90^{\circ} \mathrm{C}^{7}$, com ciclos curtos; baixo consumo de água, energia e detergente; lavagem com 12 jatos, sendo quatro com função de rotação; ciclo padrão de cinco minutos, consumo de 13 litros de água fria e 13 litros de água quente no ciclo padrão.

Para a preparação das amostras, foram inoculados $100 \mathrm{~mL}$ de água peptonada estéril no interior, passando por toda a superfície interna de cada US após cada processo realizado no expurgo. Deste volume, foram recuperados $50 \mathrm{~mL}$ e colocados no frasco original com uma seringa estéril. As amostras foram identificadas, acondicionadas e encaminhadas ao Laboratório de Microbiologia.

No Laboratório de Microbiologia, foi utilizado o método quantitativo para determinação da carga microbiana (bactérias e fungos viáveis), a técnica chamada de Spread Plate nos US ${ }^{8}$. O valor de referência aceitável é de $1 \times 10^{2} \mathrm{UFC} / \mathrm{mL}$. O Agar de Soja e Tripticaseína (TSA) e o Ágar Sabouraud com Cloranfenicol (SAB) foram utilizados para os cultivos de bactérias e fungos, respectivamente. As amostras inoculadas em TSA foram incubadas à temperatura $(\mathrm{T})$ de $32,5^{\circ} \mathrm{C} \pm 2,5^{\circ} \mathrm{C}$ por de 3 a 5 dias e $\mathrm{SAB}$ à $\mathrm{T}$ de $22,5^{\circ} \mathrm{C} \pm 2,5^{\circ} \mathrm{C}$ por de 5 a 7 dias. As leituras foram realizadas a cada 24 horas; os resultados reportados nas Fichas de Trabalho (FT) e, posteriormente, digitados no sistema e liberados. Para a expressão dos resultados, utilizou-se UFC por mL. No método qualitativo, foram isolados microrganismos de importância epidemiológica.

No método qualitativo, utilizou-se a inoculação de 1,0 mL de amostra, contendo água peptonada em frascos com $100 \mathrm{~mL}$ de Caldo de Soja e Tripticaseína (TSB) estéril. As amostras foram incubadas em estufa à $\mathrm{T}$ de $32,5^{\circ} \mathrm{C} \pm 2,5^{\circ} \mathrm{C}$ por de 3 a 5 dias. As leituras foram realizadas diariamente; se observada turbidez aparente no caldo TSB, realizou-se o isolamento dos possíveis microrganismos em meios de cultura seletivos sólidos (para bactérias gram-negativas e gram-positivas), com incubação em $32,5^{\circ} \mathrm{C} \pm 2,5^{\circ} \mathrm{C}$ durante 24 e 48 horas. Se houve crescimento no período, procedeu-se à identificação do microrganismo e foi realizado teste de sensibilidade aos antimicrobianos (TSA) para o grupo dos carbapenêmicos (Imipenem, Meropenem e Ertapenem). Se houvesse resistência plena aos carbapenêmicos, o TSA seria repetido com a bateria de antibióticos padronizados na instituição. 
$\mathrm{Na}$ fase qualitativa, os fungos não foram identificados por serem de etiologia saprófita, exceto se houvesse crescimento de elementos leveduriformes, o que não ocorreu.

O projeto foi submetido ao Comitê de Ética e Pesquisa (CEP) do hospital e aprovado conforme CAAE 64628217.3.0000.5335.

\section{RESULTADOS}

As culturas microbiológicas realizadas nos US apresentaram diferentes resultados. A Tabela 1 , com resultados da limpeza automatizada, mostra $<01$ UFC em todas as amostras analisadas.
Na limpeza automatizada, não houve crescimento de bactérias e fungos viáveis em nenhuma das 11 amostras analisadas.

Os resultados dos métodos de limpeza manual de rotina sem e com supervisão, respectivamente, contidos na Tabela 2, apresentaram isolados não detectáveis e detectáveis. Das 11 amostras analisadas, sete foram positivas para bactérias de relevância epidemiológica e dessas foram isolados 12 Bacilos Gram-Negativos (BGN), sendo sete da família Enterobacteriaceae e cinco da família dos BGN não fermentadores.

Em relação à carga microbiana, pode-se verificar que, das 11 amostras, seis tiveram a contagem no intervalo de $<01 \mathrm{UFC} / \mathrm{mL}$ a $630 \mathrm{UFC} / \mathrm{mL}\left(6,3 \times 10^{2}\right)$, e nas amostras um e dois foram isolados BGN.

Tabela 1. Métodos de lavagem/limpeza, contagem de bactérias e fungos viáveis (UFC/mL).

\begin{tabular}{|l|c|c|c|c|c|c|}
\hline \multirow{2}{*}{ Amostra } & \multicolumn{2}{|c|}{ Limpeza Automatizada } & \multicolumn{2}{c|}{ Limpeza Manual (S/S) } & \multicolumn{2}{c|}{ Limpeza Manual (C/S) } \\
\cline { 2 - 8 } & Bactérias & Fungos & Bactérias & Fungos & Bactérias & Fungos \\
\hline 1 & $<01$ & $<01$ & 50 & 41 & 15.000 & 14.000 \\
\hline 2 & $<01$ & $<01$ & 630 & 47.000 & 19 & 12 \\
\hline 3 & $<01$ & $<01$ & $<01$ & 40 & 44 & 39 \\
\hline 4 & $<01$ & $<01$ & 1680 & 40 & 375 & 91 \\
\hline 5 & $<01$ & $<01$ & 124.000 & 33.200 & 154 & 20 \\
\hline 6 & $<01$ & $<01$ & 130 & 30 & 327 & 208 \\
\hline 7 & $<01$ & $<01$ & 162 & 155.000 & 216.000 & 12.000 \\
\hline 8 & $<01$ & $<01$ & 90 & 20 & 65.000 & 37.000 \\
\hline 9 & $<01$ & $<01$ & 770.000 & 200.000 & 3.000 .000 & 127.000 \\
\hline 10 & $<01$ & $<01$ & 2.860 .000 & 380.000 & 143.000 & 33.000 \\
\hline 11 & $<01$ & $<01$ & 141.000 & 108.000 & 3.000 .000 & 37.000 \\
\hline
\end{tabular}

S/S: sem supervisão; C/S: com Supervisão.

Tabela 2. Métodos de lavagem manual e bactérias isoladas.

\begin{tabular}{|l|c|c|c|c|c|c|}
\hline \multirow{2}{*}{ Amostra } & \multicolumn{3}{|c|}{ Limpeza Manual (S/S) } & \multicolumn{3}{c|}{ Limpeza Manual (C/S) } \\
\cline { 2 - 7 } & Isolado 1 & Isolado 2 & Isolado 3 & Isolado 1 & Isolado 2 & Isolado 3 \\
\hline 1 & P. aeruginosa & ND & ND & Pseudomonas sp. & K. pneumoniae & ND \\
\hline 2 & E. cloacae & ND & ND & E. cloacae & S. marcescens & ND \\
\hline 3 & ND & ND & ND & P. putida & ND & ND \\
\hline 4 & E. cloacae & ND & ND & E. cloacae & Pseudomonas sp. & ND \\
\hline 5 & E. cloacae & Pseudomonas sp. & P. putida & Pseudomonas sp. & ND & ND \\
\hline 6 & ND & ND & ND & P. putida & ND & ND \\
\hline 7 & ND & ND & ND & E. cloacae & ND & ND \\
\hline 8 & ND & ND & ND & E. cloacae & Pseudomonas sp. & ND \\
\hline 9 & E. cloacae & S. marcescens & ND & E. cloacae & Enterobacter sp. & Pseudomonas sp. \\
\hline 10 & K. oxytoca & P. aeruginosa & ND & P. putida & ND & ND \\
\hline 11 & K. oxytoca & P. aeruginosa & ND & K. oxytoca & P. aeruginosa & ND \\
\hline
\end{tabular}

S/S: sem supervisão; C/S: com supervisão; ND: não detectável. 
Da família Enterobacteriaceae, foram isoladas as espécies de relevância epidemiológica, Enterobacter cloacae; quatro isolados foram resistentes ao Meropenem, um isolado foi resistente ao Ertapenem e não houve resistência para Imipenem. Não houve resistência aos carbapenêmicos para Klebsiella oxytoca (dois isolados) e Serratia marcescens (um isolado). Nas cinco amostras restantes, com quantificação entre $1.680\left(1,6 \times 10^{3}\right)$ e $2.860 .000(2,9 \times 10) \mathrm{UFC} / \mathrm{mL}$, foram isolados BGN com relevância epidemiológica.

Nos resultados do método de limpeza manual com supervisão, demonstrados na Tabela 2, verificou-se que, das 11 amostras analisadas, tiveram crescimento para 17 BGN, oito da família Enterobacteriaceae e nove da família dos BGN não fermentadores.

Das espécies isoladas, um Enterobacter sp. e quatro amostras de Enterobacter cloacae, dois isolados tiveram sensibilidade intermediária para Imipenem e Meropenem; um isolado resistente e um isolado com resistência intermediária a Ertapenem. Não houve resistência aos carbapenêmicos para os isolados de Klebsiella pneumoniae (um isolado), Klebsiella oxytoca (um isolado) e Serratia marcescens (um isolado).

Do grupo dos BGN não fermentadores, foram isolados cinco Pseudomonas sp., três Pseudomonas putida e um Pseudomonas aeruginosa e não houve resistência aos carbapenêmicos.

Em relação à carga microbiana das 11 amostras, cinco tiveram a contagem no intervalo de $19(1,9 \times 10)$ a $375\left(3,7 \times 10^{2}\right)$ $\mathrm{UFC} / \mathrm{mL}$, e nas amostras 2, 3, 4, 5 e 6 foram isolados BGN.

Nas seis amostras $(54,5 \%)$ restantes, com quantificação entre $1.500(1,5 \times 10)$ e $3.000 .000(3,0 \times 10) \mathrm{UFC} / \mathrm{mL}$, foram isolados $\mathrm{BGN}$ com relevância epidemiológica.

\section{DISCUSSÃO}

O presente estudo sugere benefício na utilização da limpeza automatizada para US usados nas eliminações de fezes e urina de pacientes dependentes. Identifica-se como limitação do estudo o desconhecimento do grau de contaminação dos US antes do processo de higienização e de descontaminação a serem realizados. Entretanto, como as fezes possuem o maior número de UFC por grama e todos os US testados tinham fezes inicialmente, essa limitação torna-se pouco relevante, já que, após o processo automatizado, a contagem microbiana (bactérias e fungos viáveis) foi $<01 \mathrm{UFC} / \mathrm{mL}$ em todas as amostras. A redução da carga microbiana é uma preocupação identificada por outros autores pelo impacto positivo nas IRAS após a limpeza de materiais que entram em contato com o paciente ${ }^{9,10,11}$.
Os microrganismos encontrados diferem de um estudo francês no qual, em 25 lavadoras automáticas, a maioria encontrada foi de bactérias gram-positivas, Staphylococcus sp., além de outros $\mathrm{BGN}^{4}$ em menor número. No presente estudo, na limpeza automatizada não foram detectados e nas lavagens manuais foram encontrados BGN.

Outro aspecto a ser discutido refere-se ao fato do processo ser ou não supervisionado. Poderia ser esperado que o fenômeno de Hawtorne ocorresse durante a limpeza e desinfecção supervisionada; entretanto, esse fato não ocorreu. Embora o número amostral tenha sido insuficiente para testes de significância estatística, evidenciou-se, independentemente de supervisão ou não, que o método automatizado trouxe segurança no manuseio dos US pelos profissionais, permitindo o reuso seguro entre diferentes pacientes. Outros autores comprovaram o impacto da limpeza ambiental na redução das IRAS ${ }^{10}$. Essa observação sugere analogamente que a limpeza de US teria igual impacto, já que entra em contato direto com o paciente, como no presente estudo. Da mesma forma, as recomendações de experts sobre fômites infectados têm sido motivo de discussões recentes com relação ao impacto no ambiente e nas IRAS ${ }^{11}$.

As excreções que contêm a microbiota modificada dos pacientes são eliminadas e contaminam o ambiente, modificando gradualmente a microbiota hospitalar. Materiais e superfícies contaminados com microrganismos modificados entram em contato com outros pacientes, infectando-os ou colonizando-os, criando, assim, uma cadeia interminável ${ }^{12}$. A detecção de diferenças entre os isolados identificados comprova que os US são contaminados por eles, expondo os usuários tanto aos isolados resistentes quanto os intermediários e os sensíveis aos antimicrobianos. Foi identificado por outros autores que a intervenção em higiene de fômites de pacientes reduz a disseminação de microrganismos resistentes ${ }^{13}$.

\section{CONCLUSÃO}

Com base nos resultados do presente estudo, conclui-se que o método automatizado demonstrou ser seguro para utilização dos Utensílios Sanitários na assistência à saúde. Os resultados obtidos no método manual não conferem segurança. Sugere-se que sejam realizados estudos com conhecimento prévio do grau de contaminação controlada por meio de cepas de referência. 


\section{REFERÊNCIAS}

1. Rutala WA, Weber DJ. Disinfection, sterilization, and antisepsis: an overview. Am J Infect Control [Internet]. 2016 [acessado em 10 abr. 2019];44(5 Supl.):e1-6. Disponivel em: https://doi.org/10.1016/j. ajic.2015.10.038

2. Groa E, Wohl RL, Coeperthwaite L, Beaty K, Jones K, Ladny C. Association of periOperative Registered Nurses (AORN). Sterilization and disinfection. In: Guidelines for Perioperative Practice. Denver: AORN; 2018. p. 957-83.

3. Kulkarni K, Kaczorowski D, Bonkowski A, Kovach S, Basile R. Safe tohandle? Comparing manually and machine-washed medical devices. Biomed Instrum Technol [Internet]. 2016 [acessado em 10 abr. 2019];50(s2):18-22. Disponível em: https://doi.org/10.2345/08998205-50.s2.18

4. Bros A, Deboscker S, Mielcarek M, Foeglé J, Hernandez C, Ménard $\mathrm{C}$, et al. Bacteriological quality evaluation of bed pans in a university hospital. Int J Infec Control [Internet]. 2018 [acessado em 10 abr. 2019];14(1):1-6. Disponível em: http://www.ijic.info/article/view/17934

5. Jandhyala SM, Talukdar R, Subramanyam C, Vuyyuru H, Sasikala M, Reddy DN. Role of the normal gut microbiota. World J Gastroenterol [Internet]. 2015 [acessado em 10 abr. 2019];21(29):8787-803. Disponível em: https://www.ncbi.nlm.nih.gov/pmc/articles/PMC4528021/

6. Sociedade Brasileira de Enfermeiros de Centro Cirúrgico, Recuperação Anestésica e Centro de Material e Esterilização (SOBECC). Diretrizes de práticas em enfermagem cirúrgica e processamento de produtos para a saúde. 7a ed. São Paulo: SOBECC/Barueri: Manole; 2017.

7. International Organization for Standardization. ISO 15883: Washer disinfectors [Internet]. Genebra: ISO; 2006 [acessado em 10 abr. 2019]. Disponível em: https://www.iso.org/standard/41076.html
8. United States Pharmacopeia. $37^{\mathrm{a}}$ ed. Rockville: United States Pharmacopeia I Convention; 2014.

9. Ramphal L, Suzuki S, McCracken IM, Addai A. Improving hospital staff compliance with environmental cleaning behavior. Proc (Bayl Univ Med Cent) [Internet]. 2014 [acessado em 10 abr. 2019];27(2):8891. Disponível em: https://www.ncbi.nlm.nih.gov/pmc/articles/ PMC3954653

10. Everett BR, Sitton JT, Wilson M. Efficacy and cost-benefit analysis of a global environmental cleaning algorithm on hospital-acquired infection rates. J Patient Saf. [Internet]. 2017 [acessado em 10 abr. 2019];13(4):207-10. Disponível em: https://doi.org/10.1097/ PTS.0000000000000141

11. Quinn MM, Henneberger PK, Braun B, Delclos GL, Fagan K, Huang $\mathrm{V}$, et al. A. Cleaning and disinfecting environmental surfaces in healthcare: toward an integrated framework for infection and occupational illness prevention. Am J Infect Control [Internet]. 2015 [acessado em 10 abr. 2019];43(5):424-34. Disponível em: https:// www.ajicjournal.org/article/S0196-6553\%2815\%2900075-9/pdf. http://dx.doi.org/10.1016/j.ajic.2015.01.029

12. Hoefel HH, Lautert L, Schmitt C, Soares T, Jordan S. Vancomycin administration: mistakes made by nursing staff. Nurs Stand [Internet]. 2008 [acessado em 10 abr. 2019];22(39):35-42. Disponível em: https:// doi.org/10.7748/ns2008.06.22.39.35.c6567

13. Datta R, Platt R, Yokoe DS, Huang SS. Environmental cleaning intervention and risk of acquiring multidrug-resistant organisms from prior room occupants. Arch Intern Med [Internet]. 2011 [acessado em $10 \mathrm{abr}$. 2019];171(6):491-4. Disponível em: https://jamanetwork.com/journals/jamainternalmedicine/ article-abstract/226851 